\title{
PROYECTO DE ENERGIA SOLAR PARA UN EDIFICIO
}

\section{José Scott* \\ Máximo Campusano*}

RESUMEN

Hoy día, en los comienzos del siglo XXI, más de 3,000 millones de personas viven en las zonas rurales de paises subdesarrollados sin acceso a las redes eléctricas y con pocas esperanzas de cambiar esta realidad debido a las carencias en que viven.

El desarrollo de nuevas tecnologías basadas en fuentes renovables de energía como: las energías solar, élica y de biomasa auguran un cambio en la situación eléctrica de las zonas rurales en los países tercermundistas en vista de que se pueden desarrollar sistemas energéticos aislados que no están conectados a la red eléctrica nacional con diferentes aplicaciones tales como: iluminación, bombeo de agua, cocción de alimentos y procesamientos industriales que permitirán un desarrollo sostenible a estas regiones deprimidas del planeta.

República Dominicana ya acumula una experiencia en la electrificación rural por medio de paneles fotovoltaicos que transforman la energía de la radiación solar en energía eléctrica. En la actualidad se han electrificado más de 1,500 viviendas ${ }^{1}$, unas 60 clínicas $^{2}$ y más de 25 escuelas rurales ${ }^{3}$ con paneles fotovoltaicos, lo que da una idea de la importancia del aprovechamiento de la energía solar en países en vías de desarrollo.

PALABRAS CL.AVES

Eneregia Renovable, Electricidad, Celdas Fotovoltaicas, Energía Solar

\footnotetext{
(*) Área de Ciencias Básicas y Ambientales - INTEC

Proyecto PROLINO en la Línea Noroeste, República Dominicana.

Proyecto PRISA en el sureste de la República Dominicana

Proyecto PRIDEP de la Comunidad Europea.
} 


\section{OBJETIVOS DEL PROYECTO}

Los objetivos del proyecto que nos ocupa son la alimentación eléctrica para fines esencialmente demostrativos y de estudio del edificio Pedro Bonó del Instituto Tecnológico de Santo Domingo utilizando un arreglo de paneles fotovoltaicos con los correspondientes dispositivos que completan el sistema eléctrico como baterías, inversor y reguladores. La idea es la de satisfacer todas las necesidades energéticas del edificio, en donde hay 4 oficinas y 5 aulas exceptuando el enfriamiento del aire.

A continuación describimos y desarrollamos cada uno de los pasos del diseño de un sistema eléctrico fotovoltaico para el edificio ya citado.

\section{METODOLOGÍA UTILIZADA}

Son cinco los pasos básicos a desarrollar con la finalidad de diseñar un sistema fotovoltaico independiente como el que nos ocupa:

1. Necesidades energéticas. En este paso se determina la carga a suplir y durante cuánto tiempo al día. A partir de esta información se calcula la energía que debe producir el arreglo solar para satisfacer la carga de interés.

2. Consideraciónes de las pérdidas energéticas. En este segundo paso se cuantifican las pérdidas energéticas y así obtener una carga energética más real.

3. Radiación media diaria sobre la región de interés. Se recopila información suficiente por un período de tiempo considerable sobre los niveles de radiación en la zona en donde se ubicará el arreglo solar, con la finalidad de definir la potencia de radiación de diseño.

4. Dimensionamiento del arreglo solar. Se determina el número de paneles solares a utilizar y su distribución. 
5. Dimensionamiento del banco de baterias. Se calcula el número de baterías a utilizar y su distribución.

\section{NECESIDADES ENERGÉTICAS}

Tabla para el Càlculo del Consumo de Energía

\begin{tabular}{|c|l|c|c|c|}
\hline & & & \multicolumn{1}{|c|}{$\begin{array}{c}\text { Horas } \\
\text { de } \\
\text { Cantidad }\end{array}$} & $\begin{array}{c}\text { Consumo } \\
\text { total en } \\
\text { KWh/dia }\end{array}$ \\
\hline 103 & Dispositivo & Potencia (W) & Consumo/dia & \\
& fluorescentes & 40 & 10 & 41.2 \\
19 & Bombillas PLC & 20 & 7 & 2.66 \\
22 & Computadoras & 156 & 7 & 24.02 \\
11 & Impresoras & 12 & 7 & 0.924 \\
\hline Total & & & & 68.80 \\
\hline
\end{tabular}

La energía total consumida durante un mes ( 20 días) es igual a:

$$
\begin{aligned}
20 \text { días } \times \text { consumo diario (en wh) } & =\text { Consumo Total por mes } \\
20 \times 68,800 & =1,376,000 \mathrm{wh}
\end{aligned}
$$

Se convierte este consumo en amperes-hora dividiendo el consumo total entre 48 cuando se utilizan baterías a $48 \mathrm{~V}$ :

$$
\begin{gathered}
P=V I \\
I=P / V \\
1,376,000 \div 48 \quad=28,666.7 \text { amperes-hora. }
\end{gathered}
$$

Esto implica que el arreglo solar debe producir 28,666.7 amperes-hora cada mes, lo que equivale a 1,433.3 amperes-hora por día. 


\section{CONSIDERACIÓN DE LAS PÉRDIDAS ENERGÉTICAS}

Debido a las pérdidas energéticas en el cableado e interconexiones, así como en los procesos de carga y descarga de las baterías, se considera un factor de 1.5 para obtener una carga energética más real:

Consumo diario del sistema:

$$
\begin{aligned}
68.80 \mathrm{KWh} \times 1.5 & =103.20 \mathrm{KWh} \text { por día } \\
& =103,200 \mathrm{Wh} \text { por día }
\end{aligned}
$$

\section{RADIACIÓN MEDIA DIARIA SOBRE LA REPÚBLICA DOMINICANA}

A continuación se muestra la radiación media diaria, tanto sobre un plano horizontal, como sobre un plano inclinado a $25^{\circ}$ que se recibe sobre la República Dominicana:

\section{Radiación Diaria Media Mensual en la República} Dominicana $\left(\mathbf{K W h} / \mathbf{m}^{2}\right)^{4}$

\begin{tabular}{|r|cccccccccccc|}
\hline Incl & Ene & Feb & Mar & Abr & May & Jun & Jul & Ago & Sep & Oct & Nov & Dic \\
\hline $0^{\circ}$ & 4.45 & 4.97 & 5.58 & 6.17 & 6.00 & 6.17 & 6.10 & 6.00 & 5.64 & 5.03 & 4.50 & 4.10 \\
\hline $25^{\circ}$ & 5.59 & 5.77 & 5.87 & 5.84 & 5.27 & 5.20 & 5.24 & 5.49 & 5.68 & 5.61 & 5.51 & 5.26 \\
\hline
\end{tabular}

A partir de estos resultados, asumimos el menor promedio mensual:

\section{$5.20 \mathrm{KWh} / \mathrm{m}^{2} / \mathrm{día}$}

como radiación solar de diseñ் que permitirá satisfacer las necesidades energéticas incluso durante el mes más crítico.

4 Solar Radiation Data Manual for Flat-Plate and Concentration Collectors. NREL/ TP-463-
5607 


\section{DIMENSIONAMIENTO DEL ARREGLO SOLAR}

Las dimensiones del arreglo solar se obtienen dividiendo los requerimientos diarios de energía entre la radiación solar diaria del lugar en donde se instalará el arreglo.

Para nuestro caso los cálculos son los siguientes:

$103,200 / 5.20=19,846 \mathrm{~W}=19.846 \mathrm{~kW}$

\section{ARREGLO DE PANELES SOLARES}

Es recomendable desarrollar este proyecto en cuatro etapas, cada una de $5 \mathbf{K w}$. Para cada una de estas etapas de $5 \mathrm{Kw}$ se utilizan paneles solares de $100 \mathrm{~W}$ cada uno a $12 \mathrm{~V}$ y con corriente promedio de $5.6 \mathrm{~A}$. En vista de que se utiliza un inversor con voltaje de entrada de $48 \mathrm{~V}$, se obtiene la corriente total del arreglo dividiendo la potencia requerida de $5,000 \mathrm{~W}$ entre el voltaje de trabajo (48 V)

\section{$5,000 \mathrm{~W} / 48 \mathrm{~V}=104.17 \mathrm{~A}$}

Esta intensidad de corriente a $48 \mathrm{~V}$ se obtiene con 19 líneas de paneles solares conectadas en paralelo, cada una de 4 paneles. En vista de que cada panel tiene $1.498 \mathrm{~m}$ de largo y $0.594 \mathrm{~m}$ de ancho, este arreglo solar ocupa un área de:

$$
A=6 \mathrm{~m} \times 15 \mathrm{~m}=90 \mathrm{~m}^{2}
$$

\section{DIMENSIONAMIENTO DEL BANCO DE}

\section{BATERIAS}

Las baterías de un arreglo solar tienen una larga vida si son descargadas hasta un $20 \%$ de su capacidad total, en lugar de un $80 \%$ como ocurre en las descargas profundas. Esto implica que la capacidad del banco de baterías es 5 veces mayor que la capacidad de todo el sistema. Los cálculos son los siguientes: 


\section{3,200 Wh de consumo diario Multiplicado \\ por $5=516,000 \mathrm{Wh}$}

Esta es la capacidad del banco de baterías para el proyecto completo. Si se considera un sistema modular constituido por 4 módulos: uno para cada oficina del edificio Pedro Bonó resulta que la capacidad del banco de baterías para cada módulo es igual a:

$$
516,000 / 4=129,000 \mathrm{Wh}
$$

Esta capacidad se expresa normalmente en amperes-hora, dividiendo este último resultado entre el voltaje del banco de baterías que se asume igual a $48 \mathrm{~V}$ :

$$
129,000 / 48=2,687.5 \text { amperes }- \text { hora }
$$

\section{ARREGLO DE BATERIAS}

Para el arreglo de baterías se utilizan baterías de ciclo profundo de 395 A a $6 \mathrm{~V}$. En vista de que el voltaje del banco de baterías alimenta un inversor de $48 \mathrm{~V}$ se organizan 7 filas conectadas en paralelo de baterías, en donde cada fila contiene 8 baterías.

Este arreglo ocupa un área igual de:

$$
A=1.3 \mathrm{~m} \times 2.36 \mathrm{~m}=3.068 \mathrm{~m}^{2}
$$

\section{COMPARACIÓN CON EL SISTEMA TRADICIONAL ${ }^{5}$}

Las necesidades del sistema a alimentar eléctricamente son de:

\subsection{0kwh por día}

\footnotetext{
s “Annual Energy Review" 1996 (Washington, D.C. : DOE/EIA-0384(96), July 1997).
} 


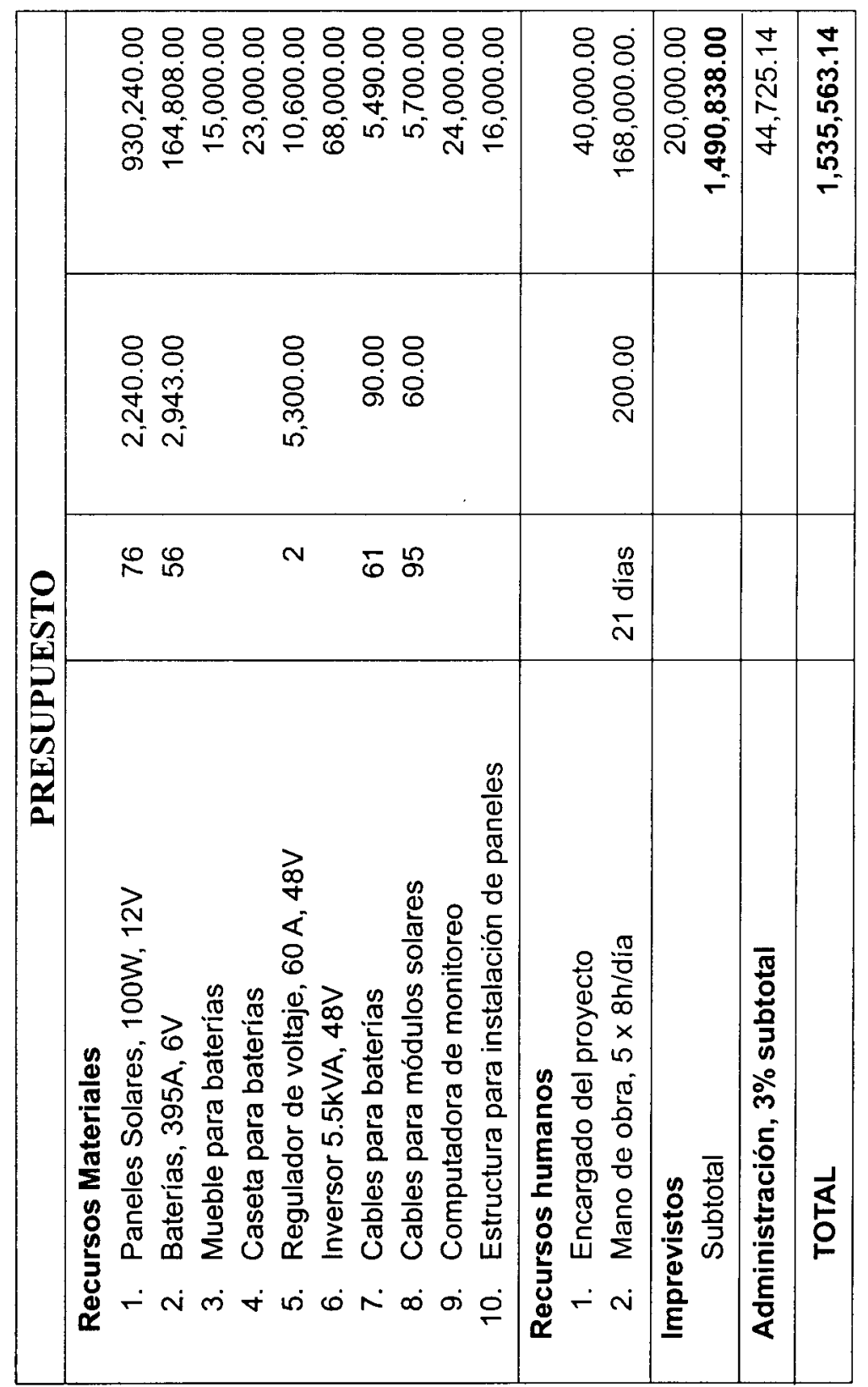


En la primera etapa del proyecto sólo se cubrirá una cuarta parte de esta necesidad.

103.20 kwh por día/ $4=25.8$ kwh por día

Al cabo de 25 años nuestro sistema fotovoltaico aportará una energía de:

$25.8 \mathrm{kwh} \times 312$ dias $\times 25$ años $=201,240 \mathrm{kwh}$

Al dividir el costo total de la 1era parte del proyecto, considerando 2 cambios de baterías de RD\$ 329,616.00 pesos, entre la energía total producida se obtiene el costo real por cada kwh del sistema fotovoltaico:

\section{$\operatorname{RD} \$ 1,865,179.14 / 201,240 \mathrm{kwh}=\operatorname{RD} \$ 9.27 / \mathrm{kwh}$}

$\mathrm{Al}$ comparar este costo por cada kwh de energía fotovoltaica con el costo máximo que se paga por la energía eléctrica de origen fósil que es de: RD\$ 2.56/ $\mathbf{k w h}$ se puede apreciar que todavía la energía solar fotovoltaica es mucho más cara que la de origen fósil. Ahora bien, en vista de que no hay ninguna contaminación provocada por la energía solar y en cambio, la producción de energía eléctrica equivalente de origen fósil emitirá a la atmósfera, al cabo de esos 25 años de utilidad que hemos considerado, los siguientes contaminantes:

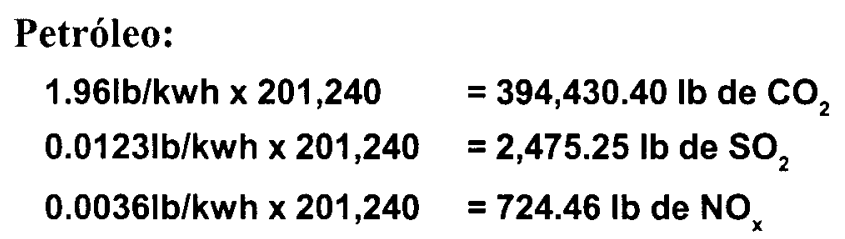

se observa que es saludable para la especie humana y para nuestro planeta, en general, que se sigan estudiando y promoviendo las fuentes de energías limpias y renovables tales como la solar y la fotovoltaica, no obstante estar en desventaja, desde el punto

\footnotetext{
6 Renewable Energy Technology Characterizations. U. S. Department of Energy and the Electric Power Research Institue, December, 1997.
} 
de vista económico, con respecto a las fuentes de energía de origen fósil ${ }^{6}$

\section{BIBLIOGRAFÍA}

1. "Annual Energy Review 1996". Washington, D. C. DOE/EIA0384(96), July, 1997.

2. "Renewable Energy Technology Characterizations". US Department of Energy and the Electric Power Research Institute. December, 1997.

3. Applied Power Corporation. "Alternative Energy". 1999 Design Guide and Catalog.

4. "Energías Renovables en la República Dominicana". Secretaría de Estado de Industria y Comercio. Santo Domingo, 2001.

5. Moreno González, Antonio. La Energía. Editorial Acento. España, 1997.

6. "Solar Radiation Data Manual for Flat-Plate and Concentration Collectors", NREL/TP-463-5607 